\title{
Neural systems underlying lexical retrieval for sign language
}

\author{
Karen Emmorey $^{\mathrm{a}, *}$, Thomas Grabowski ${ }^{\mathrm{b}}$, Stephen McCullough ${ }^{\mathrm{a}}$, Hanna Damasio ${ }^{\mathrm{a}, \mathrm{b}}$, \\ Laura L.B. Ponto ${ }^{\mathrm{b}}$, Richard D. Hichwa ${ }^{\mathrm{b}}$, Ursula Bellugi ${ }^{\mathrm{a}}$ \\ ${ }^{a}$ Laboratory for Cognitive Neuroscience, The Salk Institute for Biological Studies, 10010 North Torrey Pines Road, La Jolla, CA 92037, USA \\ ${ }^{\mathrm{b}}$ University of Iowa, Iowa City, IA, USA
}

Received 6 May 2001; received in revised form 15 July 2002; accepted 22 July 2002

\begin{abstract}
Positron emission tomography was used to investigate whether signed languages exhibit the same neural organization for lexical retrieval within classical and non-classical language areas as has been described for spoken English. Ten deaf native American sign language (ASL) signers were shown pictures of unique entities (famous persons) and non-unique entities (animals) and were asked to name each stimulus with an overt signed response. Proper name signed responses to famous people were fingerspelled, and common noun responses to animals were both fingerspelled and signed with native ASL signs. In general, retrieving ASL signs activated neural sites similar to those activated by hearing subjects retrieving English words. Naming famous persons activated the left temporal pole (TP), whereas naming animals (whether fingerspelled or signed) activated left inferotemporal (IT) cortex. The retrieval of fingerspelled and native signs generally engaged the same cortical regions, but fingerspelled signs in addition activated a premotor region, perhaps due to the increased motor planning and sequencing demanded by fingerspelling. Native signs activated portions of the left supramarginal gyrus (SMG), an area previously implicated in the retrieval of phonological features of ASL signs. Overall, the findings indicate that similar neuroanatomical areas are involved in lexical retrieval for both signs and words.
\end{abstract}

(C) 2003 Elsevier Science Ltd. All rights reserved.

Keywords: Positron emission tomography; Deaf signers; Naming; Fingerspelling; American Sign Language (ASL)

\section{Introduction}

Signed languages provide a unique approach to understanding the neural systems that underlie language processing because of their distinct modes of production and perception. The existence of languages such as American Sign Language (ASL) allow us to ask new questions about the neural structures that underlie our conceptual knowledge and use of language. What are the consequences of language developed in the hands and for the eyes, rather than based on the vocal tract and the ear? Do these modality-determined differences influence the organization of neural areas which mediate between lexical units (signs or words) and their corresponding conceptual structures? These are the issues addressed in this study.

Previous research using positron emission tomography (PET) indicates that lexical retrieval of English words for non-unique concrete entities and for unique concrete entities (e.g. retrieving the names of individual people) depends not just on the classic language areas, but also on several

\footnotetext{
* Corresponding author. Tel.: +1-858-453-4100; fax: +1-858-452-7052.

E-mail address: emmorey@salk.edu (K. Emmorey).
}

distinct regions of higher-order association cortices within the left hemisphere [15]. Damasio et al. [15] hypothesized that these association cortices within the left hemisphere mediate between the neural regions which support conceptual knowledge and those which support the phonemic representations needed for the production of the name of the concrete entity. For example, distinct intermediary regions were engaged when naming non-unique animals, compared to naming unique individual persons. Specifically, retrieving words for animals was found to activate regions within the left ventral inferior temporal lobe; in contrast, retrieving the names of famous persons activated the left and right temporal pole (TP) (the lesion data indicated that left TP is associated with name retrieval, while right $\mathrm{TP}$ is associated with recognition; [15]). The authors proposed that these left hemisphere intermediary regions do not represent names of animals or persons in explicit form; but rather their activity serves to reconstruct the phonological structure of a given word within sensorimotor structures in left perisylvian cortex.

The lexical retrieval of an ASL sign involves the reconstruction of a manual rather than a vocal representation. Many linguists have argued that signed languages exhibit a "phonological" level of structure, despite the fact that signed 
languages are perceived visually rather than auditorily $[5,10,29,33]$. In spoken languages, words are constructed out of sounds which in and of themselves have no meaning. The words bat and pat differ only in the initial sounds which have no inherent meanings of their own. Sounds may be combined in various ways to create distinct words: bad differs from $d a b$ only in how the sounds are sequenced. Similarly, signs are constructed out of components that are themselves meaningless and are combined to create morphemes and words.

Signs are composed of four basic phonological parameters: handshape, location (place of articulation), movement, and palm orientation. Fig. 1 provides an illustration of three minimal pairs: signs that are identical except for one component, and if you substitute one component for another, it changes the meaning of the sign. The top illustration shows two ASL signs that differ only in handshape. ASL contains over 30 different handshapes, but not all sign languages share the same handshape inventory. For example, the " $t$ " handshape in ASL (the thumb is inserted between the index and middle fingers of a fist) is not used by Danish sign language. Chinese sign language contains a handshape formed with an
(A)

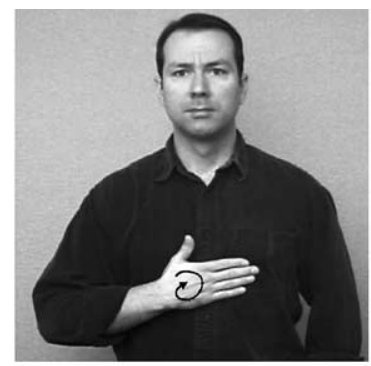

PLEASE

(B)

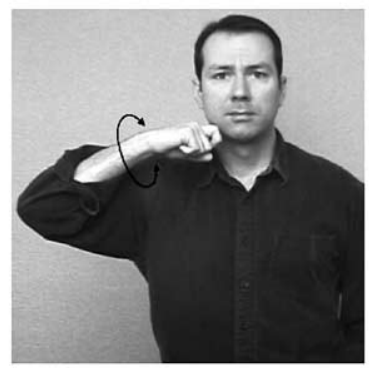

APPLE

(C)

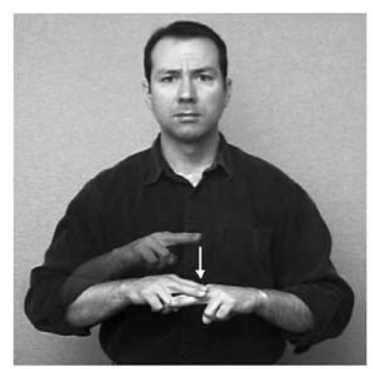

SIT

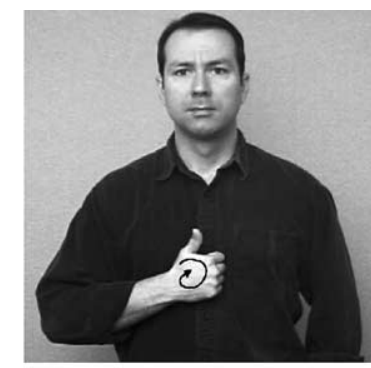

SORRY

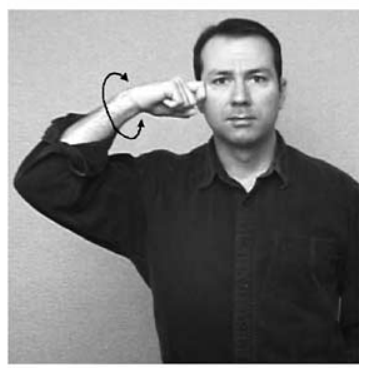

ONION

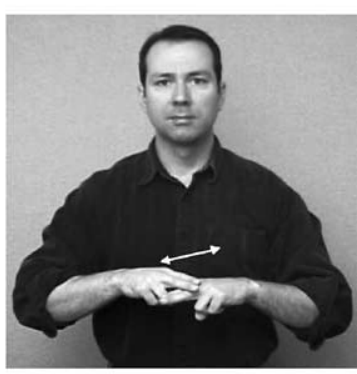

TRAIN
Fig. 1. Examples of minimal pairs in ASL: (A) signs that contrast in hand configuration; (B) signs that contrast in place of articulation, and (C) signs that contrast in movement. open hand with all fingers extended except for the ring finger which is bent-this hand configuration does not occur in ASL. Signs also differ according to where they are made on the body or face. Fig. 1B shows two signs that differ only their place of articulation. Movement is another contrasting phonological category that distinguishes minimally between signs as shown in Fig. 1C. Several different path movement types occur in ASL (e.g. circling, arc, straight), and signs can contain "internal" movement such as wiggling of the fingers or changes in handshape. Finally, signs can differ solely in the orientation of the palm; for example, the sign WANT (signs are notated as English glosses in uppercase) is produced with a spread hand with the palm up, and FREEZE is produced with the same handshape and movement (bending of the fingers and movement toward the body), but the palm is facing downward. These meaningless phonological elements are combined and sequenced to create lexical signs. Thus, both signed and spoken languages exhibit a linguistically significant, yet meaningless, level of structure which can be analyzed as phonology for both language types (see [18] for discussion).

In sum, there are several aspects of spoken language that have a direct counterpart in signed language, and nearly two decades of research on the neural organization of sign language strongly indicates that the left hemisphere is critical to linguistic functions for signers, as it is for speakers (for recent reviews see $[8,18,27])$. However, there have been few studies that investigate whether or not distinctions might exist in the neural organization of language-related systems within the left hemisphere for deaf individuals. In this study, we conducted a PET experiment with deaf ASL signers to investigate whether the retrieval of signs denoting entities belonging to two distinct conceptual categories, non-unique animals and unique persons, would engage separable regions in higher-order cortices of the left hemisphere. Given previous results with hearing English speakers, we hypothesize that left ventral inferotemporal (IT) cortex will be engaged when retrieving signs for animals and that the left temporal pole will be engaged when retrieving names for famous persons. As noted, these regions are hypothesized to represent language-related structures that mediate between cortices that support conceptual knowledge and neural structures engaged during the phonological implementation of word forms $[12,15]$.

We have no reason to suspect that deaf people have a distinct conceptualization of animals or famous persons compared to hearing people, and thus we hypothesize that similar cortices will be activated during concept retrieval and that these cortices will then engage the same intermediary language areas during lexical retrieval. The cortical regions that support concept retrieval are located in early and higher-order sensory cortices of both hemispheres, whereas language-related mediational structures are lateralized to the left hemisphere (specifically, left IT and TP for naming animals and persons, respectively). These intermediary language areas do not contain word representations; 

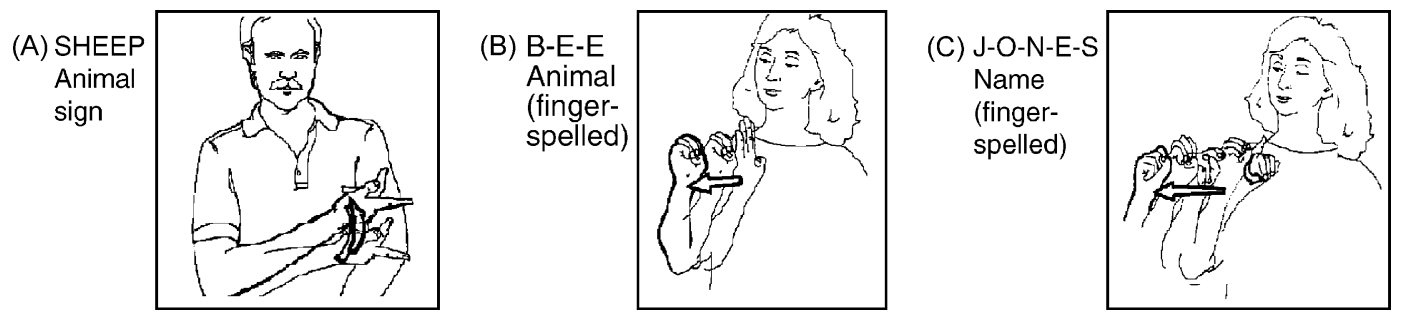

Fig. 2. Example sign responses when naming animals and persons.

rather, these cortices are hypothesized to represent disposition regions for name retrieval for entities within distinct conceptual categories, and these intermediary regions then guide phonological implementation during naming $[15,16]$.

It is possible that the neural cortices underlying phonological implementation itself differ for speech and sign. According to Levelt's model of speech production [32], phonological implementation involves phonological code retrieval, phonological encoding, phonetic encoding, and articulation. In a recent meta-analysis, Indefrey and Levelt [30] propose that the following neural regions are engaged in phonological code retrieval and encoding: left posterior and middle temporal gyri, left thalamus, left inferior frontal gyrus (IFG), and left mid superior temporal gyrus (STG). They propose that the following areas are engaged during phonetic encoding and articulation: primary motor and sensory areas, left anterior STG, supplementary motor area (SMA), and the left and medial cerebellum. This proposed neural circuitry for language production is based entirely on studies of spoken word production, and the extent to which it applies to sign language production is unclear. However, the study presented here was not designed to specifically investigate potential differences in phonological implementation for ASL compared to English; rather the study investigates those aspects of lexical retrieval that are affected by the nature of the categories to be named (i.e. higher-order association cortices within the left hemisphere).

Nonetheless, there is one important difference between English and ASL with respect to phonological implementation that is relevant to naming animals compared to naming famous persons. In English, naming these two types of entities does not involve distinct phonological processes. In contrast, for ASL, names of famous persons are almost always fingerspelled because most celebrities do not have name signs, which tend to be reserved for family, friends, and well-known deaf people. The fingerspelled alphabet is a set of signs for the English alphabet and is not a representation of the sounds of English-it is a manual representation of the orthographic representation of English. Although fluent fingerspelling clearly involves sequencing handshapes in correspondence with written letter sequences, there are constraints on the nature of movement patterns and co-articulation effects for handshape sequences [44]. Fluent and correctly articulated fingerspelling is difficult to acquire by late learners of ASL. In addition, fingerspelled names violate many of the phonological constraints on form that are observed for native ASL signs [5,35]. For example, native ASL signs allow very restricted changes in handshape, permit only a certain number of orientation changes within a single sign, and conform to constraints on which fingers may participate in handshape changes. Fingerspelled forms that violate these core phonological constraints are thus, argued to be on the periphery of the ASL lexicon [5,35].

Because of these differences between fingerspelled forms and native ASL signs, we created two sets of animal stimuli in order to compare the retrieval of lexical items denoting animals and persons: those that can be named with native ASL signs and those that must be named by fingerspelling because no ASL sign exists. Fig. 2 provides illustrations of the three types of signed responses (native ASL signs for animals, fingerspelled signs for animals, and fingerspelled names of persons). Retrieval of fingerspelled signs denoting animals can be contrasted with retrieval of fingerspelled names for famous persons and with the retrieval of native ASL signs denoting animals. We hypothesize that the cortices which mediate between phonological production of lexical items and their associated concepts will be identical for fingerspelled and native signs denoting animals, but the neural cortices responsible for phonological production itself may have a different distribution for these distinct sign forms. Specifically, we hypothesized that the left supramarginal gyrus (SMG) would be more engaged during the production of native ASL signs than during the production of fingerspelled words. This hypothesis is based on a recent cortical mapping study by Corina et al. [9] which suggested that the SMG is engaged during the selection of phonological features for native ASL signs. Corina et al. [9] found that stimulation of the left SMG in a deaf ASL signer produced both phonological and semantic errors during a naming task, and the phonological errors were clear substitutions, which differed from the reduced articulations that characterized signing under stimulation to Broca's area.

\section{Methods}

\subsection{Subjects}

Ten right-handed, adult native deaf signers were studied under a PET protocol using $\left[{ }^{15} \mathrm{O}\right]$ water. The subjects were 


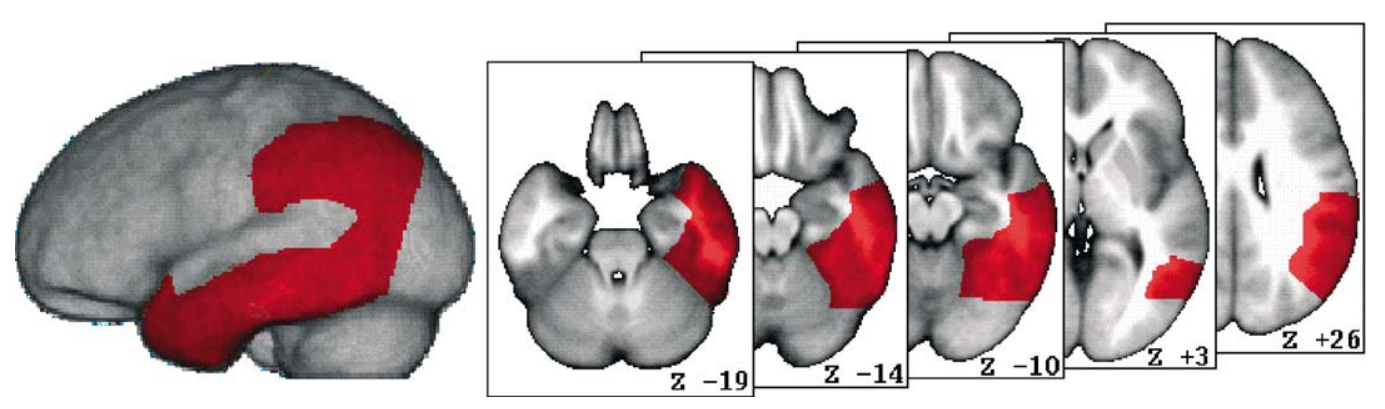

Fig. 3. A 3-D reconstructed brain showing the limits of the search volume. The search volume included voxels in Talairach space corresponding to the left temporal pole (bounded posteriorly by a line perpendicular to the major axis of the temporal lobe, at the level of the anterior ascending ramus of the sylvian fissure), left inferotemporal region (bounded by the superior temporal and collateral sulci, and the left inferior parietal lobule. The displayed axial slices correspond to the levels displayed for the results depicted in Fig. 5.

five men and five women, aged 19-38, with 12 years or more of formal education and right-handed (handedness quotient of +90 or greater as measured by the Oldfield-Geschwind questionnaire). All had deaf parents and acquired ASL as their first language from birth. All were profoundly deaf ( $90 \mathrm{db}$ loss or greater), and none had any history of neurological or psychiatric disease. All gave formal consent in accordance with Federal and institutional guidelines.

\subsection{Procedures}

All subjects underwent MR scanning in a General Electric Signa scanner operating at $1.5 \mathrm{~T}$, using the following protocol: SPGR 30, TR 24, TE 7, NEX 1, FOV $24 \mathrm{~cm}$, matrix $256 \times 192$. Each of three individual 1 NEX SPGR data sets was obtained with 124 contiguous coronal slices with thickness $1.5-1.7 \mathrm{~mm}$ and interpixel distance $0.94 \mathrm{~mm}$. The slice thickness varied so as to be adjusted to the size of the brain and the head in order to sample the entire brain, while avoiding wrap artifacts. The three individual data sets were co-registered post hoc with automated image registration (AIR 3.03) to produce a single data set, of enhanced quality, with pixel dimensions of $0.7 \mathrm{~mm}$ in plane and $1.5 \mathrm{~mm}$ between planes [28]. The MR sequences were reconstructed for each subject in 3-D using Brainvox [13,20]. Extracerebral voxels were edited away manually. The MR scans were used to confirm the absence of structural abnormalities, to plan the PET slice orientation, and to delineate regions of interest a priori.

PET-Brainvox $[14,23]$ was used to plan the PET slice orientation parallel to the long axis of the temporal lobes, so that the PET acquisition volume included the temporal lobes and the inferior parietal lobules in all subjects. Talairach space was constructed directly for each subject via user-identification of the anterior and posterior commissures and the midsagittal plane in Brainvox. An automated planar search routine defined the bounding box and a piecewise linear transformation was used [20], as defined in the Talairach atlas [39]. After Talairach transformation, the MR data sets were warped (AIR fifth-order nonlinear algorithm) to an atlas space constructed by averaging 50 normal Talairach-transformed brains, rewarping each brain to the average, and finally averaging them again (analogous to the procedure described in [43]). For simplicity, we will henceforth refer to this standard space as "Talairach space". The Talairach-transformed 3-D scans of all 10 subjects were averaged. The search volume, encompassing the left temporal pole, left inferotemporal cortices, and inferior parietal lobule, was traced on the averaged brain, so as to establish the limits and the size of the search volume (see Fig. 3).

Each subject received eight injections containing $50 \mathrm{mCi}$ of $\left[{ }^{15} \mathrm{O}\right]$ water. Each subject performed four tasks, twice each. The tasks were the following: (1) production of names for famous persons using fingerspelling (ISI $2.5 \mathrm{~s} ; N=15$ per trial); (2) production of names of animals using fingerspelling (ISI $1.5 \mathrm{~s} ; N=23$ per trial); (3) production of names of animals using native ASL signs (ISI $1.5 \mathrm{~s} ; N=23$ per trial); and (4) an orientation judgment performed on the faces of unknown persons requiring the response yes if the face was in the canonic position (up) and no if the face was inverted (ISI $1.0 \mathrm{~s} ; N=36$ per trial). The ASL signs YES and NO are fingerspelled loan signs and fall "in-between" native signs and fingerspelled words. Thus, subjects made a signed response, but no naming was involved. This task was chosen because unknown faces do not evoke a name and yet depict real entities at least as complex as the stimuli presented in the other naming tasks, and it is the task used in parallel studies with hearing English speakers [15,16,26]. Similarly, the various ISIs for each naming task were chosen to parallel the ISI times in the Damasio et al. study [15], and these times were selected to equate naming performance across tasks. Subjects' responses were recorded during the PET study by a native ASL signer, and the responses were also videotaped for confirmation and later analysis. The stimuli were presented from $5 \mathrm{~s}$ after each injection (approximately $10 \mathrm{~s}$ before the bolus arrived in the brain) until $40 \mathrm{~s}$ after injection; the average duration of a trial was $36 \mathrm{~s}$.

For all tasks, subjects responded with their right hand in a natural "whisper mode" so that the hand did not contact the face. This response mode is more natural for fingerspelling 
than for producing native signs. Therefore, subjects were always first presented with animal stimuli that could be named with native ASL signs. This presentation order reduced the possibility that subjects would fall into a fingerspelling "set" and produce fingerspelled responses in all conditions.

Face stimuli were black and white photographs, with background details and telltale appendages deleted. Familiar faces for a given subject were selected prior to the PET session by having the subjects view a large collection of famous faces from the Iowa [41] and Boston [1] Famous Faces tests, and a number of additional faces of contemporary famous actors, politicians, and sports figures. Subjects were not asked to name any of the persons, and no names were provided by the investigators. Subjects were asked to indicate whether or not they recognized each person. The final stimulus set of famous faces for each subject was composed only of faces they had said they recognized. Unfamiliar faces for the face baseline task were half male, half female, and half were inverted. Animal stimuli were digitized color photographs, from which background details had been deleted. Fig. 4 provides an illustration of each stimulus type.

Positron emission tomography (PET) data were acquired with a General Electric 4096 Plus body tomograph, yielding 15 transaxial slices with a nominal interslice interval of $6.5 \mathrm{~mm}$. For each injection, $50 \mathrm{mCi}$ of $\left[{ }^{15} \mathrm{O}\right]$ water was administered as a bolus through a venous catheter. Arterial blood sampling was not performed.

Reconstructed images of the distribution of radioactive counts from each injection were coregistered with each other using automated image registration (AIR 3.03, Roger Woods, UCLA). The 3-D MR and the mean coregistered PET data were also coregistered using PET-Brainvox and automated image registration [42]. PET data were Talairachtransformed as described above, masked to the coregistered MRI brain contour to exclude extracerebral voxels, and then smoothed with an isotropic $16 \mathrm{~mm}$ gaussian kernel by Fourier transformation, complex multiplication, and reverse Fourier transformation. The final calculated image resolution was $18 \mathrm{~mm} \times 18 \mathrm{~mm} \times 18 \mathrm{~mm}$.

PET data were analyzed with a pixelwise linear model which estimated coefficients for global flow (covariable) and task and block/subject effects (classification variables) $[21,24]$. We searched for increases in adjusted mean activity in images of $t$-statistics generated for each of the planned contrasts. Critical $t$ values were calculated using gaussian random field theory for $t$-statistics [46,47].

The planned contrasts were as follows: (a) to address the hypothesis that both naming of animals and naming of unique persons will engage, respectively, the left IT region and the left temporal pole, the standard control task (faces upright and upside-down) was subtracted from each of the target tasks; (b) to assess how the target conditions (naming of famous persons and naming of animals, both fingerspelled) would differ from each other, these target tasks were contrasted; (c) to explore the possibility that fingerspelling and signing might engage different brain regions, the two tasks naming animals were contrasted.
(A) Famous Person

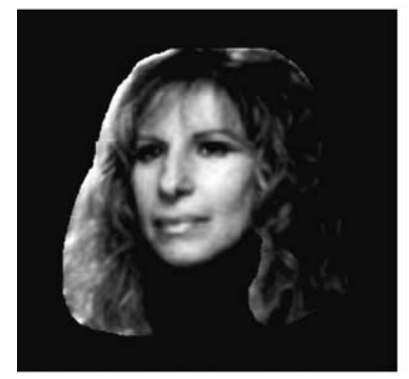

(C) Animal (native sign set)

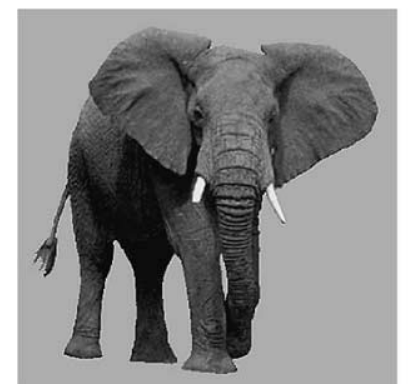

(B) Animal (fingerspelled set)

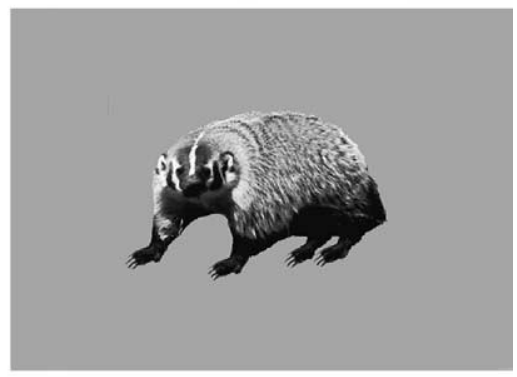

(D) Inverted unfamilar face (from the control task)

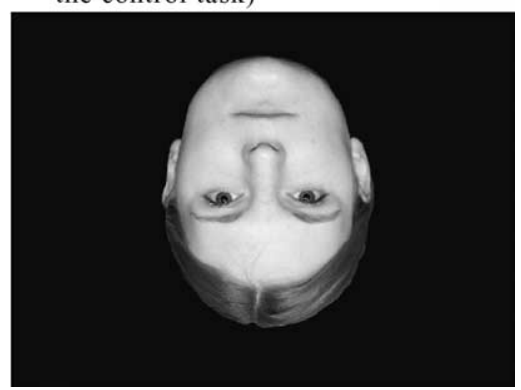

Fig. 4. Example stimuli (the pictures of animals are reproduced in black and white; they were in color for the actual study). (A) Barbra Striesand (famous person); (B) beaver (named with a fingerspelled word); (C) elephant (named with a native ASL sign); (D) an inverted unfamiliar face from the control condition. 


\section{Results}

Analysis of the behavioral data indicated that naming animals with fingerspelled signs and naming famous persons produced similar non-response rates: $17.9 \%$ for animals and $20.3 \%$ for persons. These rates are similar to the nonresponse rates observed for English speakers: $14.8 \%$ for animals and $17.0 \%$ for persons [15]. However, the non-response rate for naming animals with native ASL signs was significantly lower $(2.1 \%)$ than for the other two naming tasks $(\mathrm{F}(2,18)=9.73, P<0.01)$.

Statistically significant activity in the search volume (left inferior parietal lobule, left IT and temporal pole) with a volume of $97.4 \mathrm{~cm}^{3}$ (corresponding to 17 resels over which the critical $t$ value is \pm 3.73 ) can be seen in Table 1 and Fig. 5. To provide a direct comparison with the results from hearing English speakers, data from $[15,16,26]$ are listed in Table 3.

The contrast between naming famous persons and the control task demonstrated activation of the left temporal pole $(-38,+16,-19)$ and the left anterior superior temporal sulcus $(-53,-10,-10)$, as had been found previously for English speakers using the same post processing as this report (see Table 3; [15]: L TP $-37,+3,-19$ and L ASTS: -56 , $-14,-9$; [26]: L TP $-41+15-19)$. The contrast between naming animals (fingerspelled and signed) and the control task showed significant activation in left ventral IT $(-38$, $-45,-11)$, as predicted and as found for English speakers ([15]: $-37,-35,-15)$. Furthermore, the contrast between naming famous persons and naming animals (fingerspelled) showed significant activation in the left temporal pole $(-35$, $+12,-19)$ and left anterior superior temporal sulcus $(-50$, $-14,-10)$ during naming of famous persons relative to animals, but no difference in activity in ventral left IT during either task. Surprisingly, the contrast between naming persons and the control task also revealed activation in left ventral IT $(-40,-35,-14$; Table 1$)$, unlike what has been reported for English speakers. Finally, homologous regions within the right hemisphere were not activated when deaf signers named animals or persons, relative to the control task.

A post hoc analysis at whole brain level (critical $t=$ \pm 4.68 ) showed some additional areas of significant activity (Table 2). Naming persons and naming animals both activated the left inferior frontal gyrus, relative to the control task $(-34,+19,-3$ and $-41,+28,+17$, respectively). Similarly, activation within IFG has been reported for English speakers naming animals or persons, using the same control task ([15] for animals: $-41,+22,+3$ ). Activation within the right frontal pole was found for naming persons, relative to naming animals $(+25,+63,+8)$, but no other regions within the right hemisphere exhibited significant activation.

When the retrieval of fingerspelled words and the retrieval of native signs for animals were contrasted, there were no regions within the search volume where there was significantly more activity during retrieval of fingerspelled words. Outside the search volume, there was significant bilateral activation in superomesial frontal cortex during fingerspelling $(+1,+23,+40)$. As predicted, the retrieval of native signs

Table 1

Summary of PET activation results for deaf subjects, a priori search volume

\begin{tabular}{|c|c|c|c|c|c|}
\hline & $\mathrm{T}_{88} \mathrm{X}$ & $\mathrm{T}_{88} \mathrm{Y}$ & $\mathrm{T}_{88} \mathrm{Z}$ & $\mathrm{T}_{\max }$ & Volume $\left(\mathrm{mm}^{3}\right)$ \\
\hline \multicolumn{6}{|l|}{ Naming persons minus baseline } \\
\hline L temporal pole & -38 & +16 & -19 & 4.52 & 521 \\
\hline $\mathrm{L}$ anterior superior temporal sulcus & -53 & -10 & -10 & 4.27 & 547 \\
\hline L ventral IT & -40 & -35 & -14 & 4.43 & 2369 \\
\hline $\mathrm{L}$ angular gyrus & -31 & -74 & +39 & 4.35 & 663 \\
\hline \multicolumn{6}{|l|}{ Naming animals (fs, $\mathrm{s}$ ) minus baseline } \\
\hline $\mathrm{L}$ ventral IT & -38 & -45 & -11 & 4.59 & 16377 \\
\hline \multicolumn{6}{|l|}{ Naming persons minus animals (fs) } \\
\hline L temporal pole & -35 & +12 & -19 & 6.28 & $6974^{*}$ \\
\hline $\mathrm{L}$ anterior superior temporal sulcus & -50 & -14 & -10 & 5.74 & $6974^{*}$ \\
\hline L supramarginal gyrus & -53 & -62 & +27 & 4.20 & 684 \\
\hline \multicolumn{6}{|l|}{ Naming animals (fs) minus persons } \\
\hline L temporo-occipital junction & -39 & -62 & -5 & 4.27 & 3250 \\
\hline \multicolumn{6}{|l|}{$\begin{array}{l}\text { Naming animals (fs) minus animals (s) } \\
\text { None }\end{array}$} \\
\hline \multicolumn{6}{|l|}{ Naming animals (s) minus animals (fs) } \\
\hline \multirow[t]{2}{*}{ L lateral TO junction } & -58 & -57 & +3 & 4.04 & 415 \\
\hline & -45 & -64 & +11 & 3.92 & 192 \\
\hline L ventral IT & -42 & -9 & -14 & 3.85 & 33 \\
\hline \multirow[t]{2}{*}{ L supramarginal gyrus } & -51 & -36 & +26 & 4.16 & 639 \\
\hline & -49 & -60 & +23 & 4.08 & 10 \\
\hline
\end{tabular}

a The fingerspelled animal set is indicated by "fs" and the signed animal set is indicated by "s". An $(*)$ indicates two distinct local maxima within one suprathreshold cluster. 


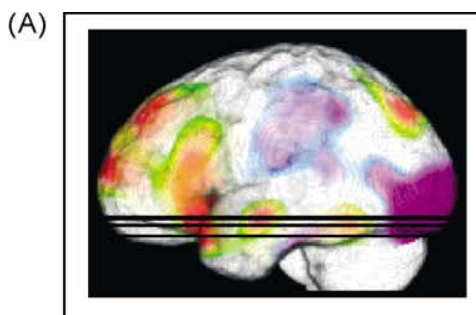

Naming persons minus control

(B)

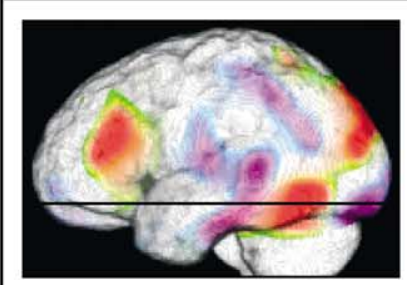

Naming animals minus control

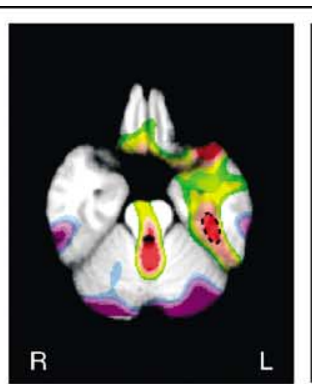

$$
Z=-19
$$

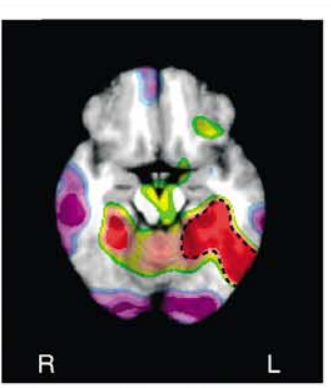

$Z=-11$

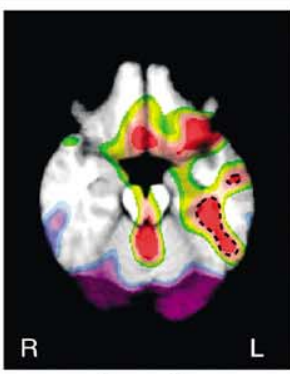

$Z=-14$

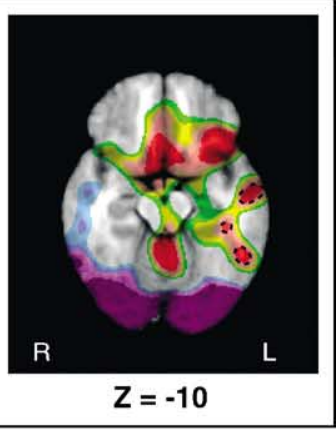

$Z=-10$

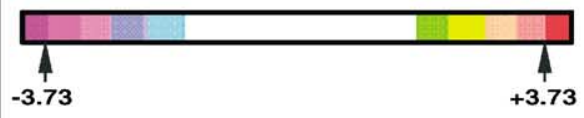

(C)

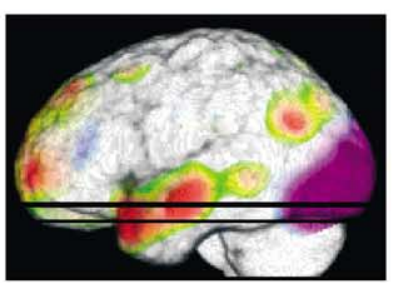

Naming persons minus naming animals
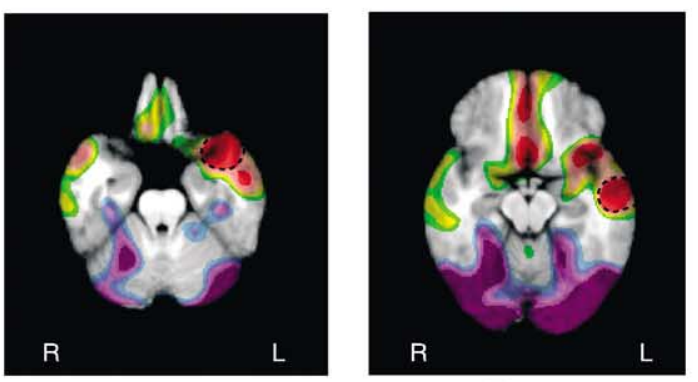

(D)

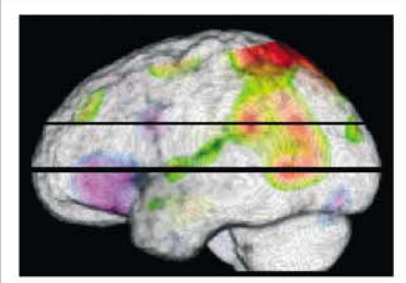

Naming animals signed minus naming animals fingerspelled

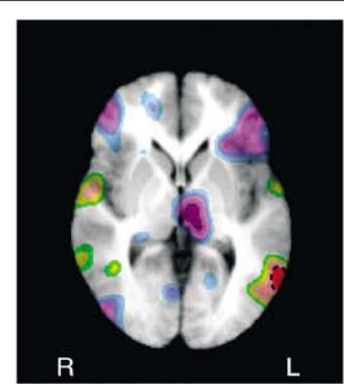

$Z=+3$

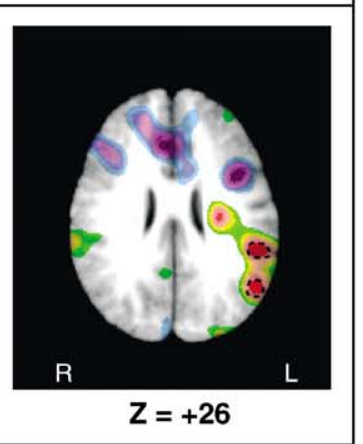

Fig. 5. Illustration of the contrast between (A) naming famous persons and the control task; (B) naming animals (fingerspelled and signed) and the control task; (C) naming famous persons and naming animals (fingerspelled); (D) naming animals with native ASL signs and naming animals with fingerspelled words. The color bar indicates the thresholds for statistical significance, corrected over the search volume of left TP, IT, and inferior parietal lobule. The black dotted line encloses those regions that are above threshold.

activated the left supramarginal gyrus relative to the retrieval of fingerspelled words. (There were two separate maxima: $-51,-36,+26$ and $-49,-60,+23$; Table 1.) There was also more activity in the left lateral temporal-occipital junction (centered at two coordinates: $-58,-57,+3$ and -45 , $-64,+11)$. Outside the search volume, there was more left superior parietal lobule activity $(-15,-59,+55)$ during the production of native ASL signs (Table 2).

\section{Discussion}

As predicted, the contrast between the control task and lexical retrieval of signs for animals revealed activation in mesial and ventral inferotemporal cortex in deaf signers, in the same region that is activated in hearing speakers when naming animals [15] (see Tables 1 and 3). The activation in these two studies was centered on coordinates $10 \mathrm{~mm}$ 
Table 2

Summary of PET activation results for deaf subjects, post hoc search volume ${ }^{\mathrm{a}}$

\begin{tabular}{|c|c|c|c|c|c|}
\hline & $\mathrm{T}_{88} \mathrm{X}$ & $\mathrm{T}_{88} \mathrm{Y}$ & $\mathrm{T}_{88} \mathrm{Z}$ & $\mathrm{T}_{\max }$ & Volume $\left(\mathrm{mm}^{3}\right)$ \\
\hline \multicolumn{6}{|l|}{ Naming persons minus baseline } \\
\hline $\mathrm{L}$ anterior cingulate & -1 & +38 & +18 & 4.89 & 8682 \\
\hline $\mathrm{L}$ inferior frontal gyrus & -34 & +19 & -3 & 7.05 & 6265 \\
\hline $\mathrm{L}$ retrosplenial & -2 & -55 & +13 & 5.04 & 1625 \\
\hline Cerebellar vermis & 0 & -47 & -12 & 5.35 & 1049 \\
\hline Ventromedial frontal & +3 & +13 & -8 & 5.10 & 597 \\
\hline L superior prefrontal & -16 & +42 & +38 & 5.76 & 423 \\
\hline Basal forebrain & +1 & 0 & -1 & 4.74 & 41 \\
\hline \multicolumn{6}{|c|}{ Naming animals (fs, s) minus baseline } \\
\hline Early visual cortex & -12 & -63 & +4 & 7.12 & 41561 \\
\hline $\mathrm{L}$ inferior frontal gyrus & -41 & +28 & +17 & 5.75 & 2383 \\
\hline L lateral occipital & -37 & -85 & +17 & 4.94 & 272 \\
\hline \multicolumn{6}{|l|}{ Naming persons minus animals (fs) } \\
\hline Mesial prefrontal & +5 & +53 & +5 & 5.28 & 3956 \\
\hline Mesial parietal & +1 & -52 & +25 & 5.75 & 2857 \\
\hline $\mathrm{L}$ anterior middle frontal gyrus & -17 & +48 & +7 & 5.34 & 772 \\
\hline Basal forebrain & +2 & +7 & -4 & 5.20 & 693 \\
\hline $\mathrm{R}$ frontal pole & +25 & +63 & +8 & 4.79 & 119 \\
\hline \multicolumn{6}{|l|}{ Naming animals (fs) minus persons } \\
\hline L temporo-occipital & -21 & -63 & 0 & 4.82 & 172 \\
\hline Bilateral early visual cortex & +2 & -80 & +2 & 4.80 & 67700 \\
\hline \multicolumn{6}{|c|}{ Naming animals (fs) minus animals (s) } \\
\hline Superomesial frontal & +1 & +23 & +40 & 5.21 & 609 \\
\hline \multicolumn{6}{|c|}{ Naming animals (s) minus animals (fs) } \\
\hline L superior parietal lobule & -15 & -59 & +55 & 5.96 & 5149 \\
\hline
\end{tabular}

a The fingerspelled animal set is indicated by "fs" and the signed animal set is indicated by "s".

apart. We have previously estimated that such coordinates are stable within a radius of $15 \mathrm{~mm} 95 \%$ of the time using these methods [24]. Therefore, the coordinate reported here is not distinguishable from that reported for English

Table 3

Summary of PET activation results for hearing subjects, a priori search volume

\begin{tabular}{|c|c|c|c|c|}
\hline & $\mathrm{T}_{88} \mathrm{X}$ & $\mathrm{T}_{88} \mathrm{Y}$ & $\mathrm{T}_{88} \mathrm{Z}$ & $\mathrm{T}_{\max }$ \\
\hline \multicolumn{5}{|l|}{ Naming persons minus baseline } \\
\hline $\mathrm{L}$ temporal pole $\mathrm{e}^{\mathrm{a}}$ & -37 & +3 & -33 & 4.57 \\
\hline L temporal pole ${ }^{\mathrm{b}}$ & -39 & +15 & -17 & 3.79 \\
\hline $\mathrm{L}$ anterior superior temporal sulcus ${ }^{\mathrm{a}}$ & -56 & -14 & -9 & 4.11 \\
\hline $\mathrm{R}$ temporal pole $\mathrm{a}^{\mathrm{a}}$ & +42 & 0 & -34 & 4.50 \\
\hline \multicolumn{5}{|l|}{ Naming animals minus baseline } \\
\hline $\mathrm{L}$ ventral $\mathrm{IT}^{\mathrm{a}}$ & -37 & -35 & -15 & 5.43 \\
\hline $\mathrm{L}$ temporal pole ${ }^{\mathrm{a}}$ & -30 & 0 & -31 & 4.36 \\
\hline \multicolumn{5}{|l|}{ Naming persons minus animals } \\
\hline $\mathrm{L}$ temporal pole $\mathrm{c}^{\mathrm{c}}$ & -39 & +3 & -17 & 3.35 \\
\hline $\mathrm{L}$ anterior superior temporal sulcus $\mathrm{c}$ & -53 & -14 & -2 & 4.55 \\
\hline \multicolumn{5}{|l|}{ Naming animals minus persons } \\
\hline $\mathrm{L}$ ventral $\mathrm{IT}^{\mathrm{c}}$ & -44 & -59 & -10 & 6.69 \\
\hline $\mathrm{L}$ ventral $\mathrm{IT}^{\mathrm{c}}$ & -20 & -66 & +3 & 5.79 \\
\hline
\end{tabular}

\footnotetext{
${ }^{a}$ H. Damasio et al. [15]

${ }^{\mathrm{b}}$ Grabowski et al. [26].

${ }^{\mathrm{c}}$ H. Damasio et al. [16].
}

speakers. The contrast between the control task and naming famous persons revealed activation in the temporal pole that was predicted, and which also falls within a $15 \mathrm{~mm}$ radius of the activation found previously for English speakers [15,24-26]. In addition, there was activation of the left anterior superior temporal sulcus, which has also been reported previously, at a coordinate $5 \mathrm{~mm}$ away from that reported here $[15,22,26]$. When we directly contrasted naming famous persons with naming animals (fingerspelled), we found significant activation of the left temporal pole and left anterior superior temporal sulcus during the task of naming famous persons, but no significant difference in activity in ventral IT between these two conditions.

These data reveal that left temporal cortices are engaged in the retrieval of both signs and words, which we interpret to mean that language modality does not affect the anatomical locus for the mediation between concept and phonological sequencing. For both signers and speakers, retrieval of lexical items denoting unique versus non-unique concrete entities (individual persons versus animals) depends upon partially segregated neural systems. These cortical regions lie outside the classical language areas and are hypothesized to contain neuron ensembles that can transiently direct the reconstruction of phonological representation (e.g. $[11,12])$. These results suggest that the modality of the phonological representation (auditory-oral or visual-manual) does not 
substantially alter the neural substrate for lexical mediation between concepts and the production of lexical items.

With respect to the production of lexical items (phonological implementation), the results of the post hoc whole brain analysis suggest some similarities between sign and speech. Specifically, left IFG was activated when naming animals relative to the baseline in $\operatorname{ASL}(-41,+28,+17)$ and in English ([15]: $-41,+22,+3)$. The homologous right hemisphere region was not activated for either speakers or signers. Indefrey and Levelt [30] propose that left IFG is one of the areas specifically involved in phonological encoding during word production. The other area hypothesized to be involved in phonological encoding is the left mid superior temporal gyrus, which was not found to be active for speakers or signers, at least with the whole brain analyses using this baseline task. Because both the target and baseline tasks involved overt articulation, these studies are unlikely to reveal neural areas involved in phonetic implementation or articulation for either speech or sign. The fact that both word and sign production engaged left IFG (specifically Broca's area) suggests that at least some of the neural circuitry involved in phonological encoding is unaffected by the nature of the articulators involved in language production. It is possible, however, that modality-related differences occur within other left perisylvian regions, given that our studies were not designed to examine the phonological implementation stage of lexical retrieval.

The contrast between naming famous persons and the control task indicated that neural regions in left ventral IT were active for deaf ASL signers (in addition to temporal pole activity; Table 1). However, activation in left ventral IT was not observed for English speakers naming famous persons [15]. It is possible that this additional activation in ventral IT is related to the different perceptual and cognitive experience that deaf signers have with the human face. Specifically, certain facial expressions are linguistic and play a significant role in the syntax and morphology of ASL, and signers must be able to discriminate rapidly among many distinct expressions during language comprehension (see [18] for a review). Furthermore, McCullough and Emmorey [34] found that deaf and hearing signers exhibited an enhanced ability to discriminate among similar faces and to recognize subtle changes in specific facial features, compared to hearing non-signers (see also [3,4]); however, ASL signers and hearing non-signers did not differ in their ability to recognize faces they had seen before or in gestalt face processing abilities. This pattern of results was attributed to the fact that to identify and categorize ASL facial expressions, signers need not recognize the person signing. Rather, signers must rely on an enhanced featural processing of the face in order to identify specific facial expressions. In addition, the gestalt of the face does not change with different facial expressions. When naming and recognizing the faces of famous persons, ASL signers engage additional neural areas in these more posterior regions perhaps because they do so whenever they process human faces. It is noteworthy that the region of activation within left IT borders the middle fusiform gyrus, an area that has been found to be engaged during part-based (featural) processing of unfamiliar faces (Rossion et al. [37] report the following co-ordinates: $-42,-50,-26$; compare to $-40,-35,-14$ for naming persons in ASL, see Table 1). Although processing the unfamiliar faces in the control condition might engage these areas, in principle, half of those faces were upside-down and thus, would not be expected to do so (e.g. [19]). Thus, we speculate that regions in ventral left IT may be automatically engaged in a more general featural analysis of faces (not specific to individuals) that is required for linguistic processing of facial expression in ASL.

The contrast between naming animals with fingerspelled signs and native ASL signs indicated significant activation in superomesial frontal cortex during fingerspelling, using the post hoc search volume. This neural region includes part of the supplementary motor area. This area has been implicated in the planning of sequences of voluntary motor movements (e.g. [40]), and unlike native ASL signs, fingerspelled words require the production of a sequence of several distinct handshapes. Furthermore, for speech production, the SMA has been implicated in the formulation of an articulatory plan and in initiating sequential articulations $[45,48]$. The additional motoric planning required by fingerspelling may therefore, have engaged the SMA to a greater extent than the production of native ASL signs, which are produced with either a single handshape or at most a sequence of two handshapes.

Naming animals with native ASL signs produced more activation in the left lateral temporal-occipital junction, compared to naming animals with fingerspelled words (Table 1). One possible explanation for this difference is that the animals in the set named with ASL signs tended to be more common animals than those animals in the fingerspelled set (the mean frequency of the English gloss for animals in the native sign set was 16.3, compared to 3.1 for the fingerspelled set, based on [31]). The behavioral data indicated that the animals in the native sign set were easier to name than the animals in the fingerspelled set. The additional activation for naming animals with native signs is quite posterior and near visual association cortices. Because more common animals are more familiar, naming these animals may not need to recruit the more anterior cortices in order to be disambiguated [12]. Naming less common animals may engage anterior regions within the ventral stream because recognition requires more analysis in order to disambiguate them from familiar animals (e.g. distinguishing a beaver from a large cat).

Finally, retrieval of native ASL signs, in contrast to fingerspelled signs for animals, produced activation in left supramarginal gyrus. As noted earlier, Corina et al. [9] found that stimulation to the left SMG in a deaf signer during a naming task caused the signer to produce phonological errors that involved mis-selections of phonological components; for example, the signer produced a clearly articulated $\mathrm{X}$ handshape (fist with index finger extended and curved) for the 
A-bar handshape (fist with thumb extended) of the intended sign PEANUT. Corina et al. [9] hypothesized that the SMG plays a critical role in the selection of phonological feature information during sign language production. Our results support this hypothesis: the supramarginal gyrus was more engaged when signers produced native ASL signs, and only these signs are represented as hierarchically arranged sets of phonological features (e.g. [5]). As noted in the introduction, fingerspelled forms are on the periphery of the ASL lexicon, violating many of the phonological constraints of native ASL signs.

Although the left SMG has been implicated in some studies of auditory phonological processing (e.g. [6,17]), there have been very few reports in which left SMG is found to be engaged during spoken word production (see [30]). It is possible that left inferior parietal cortex is uniquely involved in phonological code retrieval for ASL signs because signs, unlike fingerspelled words, require that locations on the body be specified as part of the phonological representation (see Fig. 1). Although speech requires that sounds be specified for place of articulation, the place locations are all within the vocal tract and do not have a clear spatial component. In contrast, phonologically contrastive locations for ASL signs occur on the face, head, neck, torso, arm, and hand (see [5] for a detailed list of contrasting locations); and signers must co-ordinate their hand posture to reach a particular location at the angle required to articulate the correct form of the sign. There is some evidence that inferior parietal cortex is involved in co-ordinating hand movements within body-centered space (e.g. [2,7,38]). Although the ASL signers produced whispered signs and did not produce complete movements to target body locations, they did access the location specifications for signs and often produced location distinctions within constrained "whisper space." Thus, it is possible that activation within left inferior parietal cortex (specifically, SMG) reflects an aspect of phonological implementation that is unique to signed languages.

In conclusion, we are beginning to identify and characterize the neural systems that underlie language processing in normal deaf adults who have acquired American Sign Language as their first and primary language. The data from this study indicate that the neural systems involved in the retrieval of ASL signs are comparable to those involved in retrieving spoken English words within distinct conceptual categories. Similar cortical regions within the left cerebral hemisphere mediate between recognition of a unique entity (a known individual) or a concrete non-unique entity (an animal) and the retrieval of either a spoken word or a manual sign denoting those entities. In addition, the left inferior frontal gyrus appears to play a role in phonological processing for both spoken and signed languages. However, sign production, unlike speech production, may also recruit left inferior parietal cortex (SMG). Finally, lexical retrieval does not appear to recruit additional regions within the right hemisphere for sign language, despite the visual-spatial modality. This finding is consistent with the results of lesion studies with deaf signers (e.g. [36]) and indicates that sign production, like speech production, is strongly lateralized to the left hemisphere. Overall, the results suggest that many of the neural structures that mediate language output, at least at the level of naming concrete entities, are the same regardless of the mode of output, spoken or signed.

\section{Acknowledgements}

This research was supported by a grant from the National Institute on Deafness and other Communicative Disorders, 1 P50 DC 03189, awarded to the University of Iowa and the Salk Institute for Biological Studies. We thank Kevin Clark, Brenda Falgier, Samuel Hawk, Kathy Jones, Melissa Herzig, and Jon Spradling for their help in conducting the study. We thank Ed Klima for helpful comments on a draft of the manuscript, and we particularly thank all of the Deaf subjects who participated in the study.

\section{References}

[1] Albert MS, Butters N, Levin JA. Temporal gradients in the retrograde amnesia of patients with alcoholic Korsakoff's disease. Archives of Neurology 1979;36:211-6.

[2] Andersen RA, Snyder LH, Bradley DC, Xing J. Multimodal representation of space in the posterior parietal cortex and its use in planning movements. Annual Review of Neuroscience 1997;20: 303-30.

[3] Bellugi U, O’Grady L, Lillo-Martin D, O'Grady M, Hoek K van, Corina, D. Enhancement of spatial cognition in deaf children. In: Volterra V, Erting CJ, editors. From gesture to language in hearing and deaf children. New York: Springer; 1990. p. 278-98.

[4] Bettger J, Emmorey K, McCullough S, Bellugi U. Enhanced facial discrimination: effects of experience with American Sign Language. Journal of Deaf Studies and Deaf Education 1997;2:223-33.

[5] Brentari DA. Prosodic model of sign language phonology. Cambridge (MA): MIT Press; 1998

[6] Celsis P, Boulanouar K, Doyon B, Ranjeva JP, Berry I, Nespoulous $\mathrm{JL}$, et al. Differential fMRI responses in the left posterior superior temporal gyrus and left supramarginal gyrus to habituation and change detection in syllables and tones. Neuroimage 1999;9(1): 135-44.

[7] Colby CL, Goldberg ME. Space and attention in parietal cortex. Annual Review of Neuroscience 1999;22:319-49.

[8] Corina DP. The processing of sign language: evidence from aphasia. In: Stemmer B, Whitaker HA, editors. Handbook of neurolinguistics. New York: Academic Press; 1998. p. 313-29.

[9] Corina DP, McBurney SL, Dodrill C, Hinshaw K, Brinkley J, Ojemann G. Functional roles of Broca's area and supramarginal gyrus: evidence from cortical stimulation mapping in a deaf signer. Neuroimage 1999;10:570-81.

[10] Corina DP, Sandler W. On the nature of phonological structure in sign language. Phonology 1993;10:165-207.

[11] Damasio A. Time-locked multiregional retroactivation: a systemslevel proposal for the neural substrates of recall and recognition. Cognition 1989;33:25-62.

[12] Damasio A, Damasio H. Cortical systems for retrieval of concrete knowledge: the convergence zone framework. In: Koch C, editor. Large-scale neuronal theories of the brain. Cambridge (MA): MIT Press; 1994. p. 61-74. 
[13] Damasio H, Frank R. Three-dimensional in vivo mapping of brain lesions in humans. Archives of Neurology 1992;49:137-43.

[14] Damasio H, Grabowski TJ, Frank R, Knosp B, Hichwa RD, Watkins GL, et al. PET-Brainvox, a technique for neuroanatomical analysis of positron emission tomography images. In: Uemura K, Lassen NA, Jones T, Kanno I, editors. Quantification of brain function. Amsterdam: Elsevier; 1994. p. 465-74.

[15] Damasio H, Grabowski TJ, Tranel D, Hichwa R, Damasio AR. A neural basis for lexical retrieval. Nature 1996;380:499-505.

[16] Damasio H, Tranel D, Grabowski TJ, Adolphs R, Damasio AR. Neural systems behind word and concept retrieval, Cognition [in press].

[17] Démonet J-F, Chollet F, Ramsay S, Cardebat D, Nespoulous JL, Wise R, et al. The anatomy of phonological and semantic processing in normal subjects. Brain 1992;115:1753-68.

[18] Emmorey K. Language, cognition, and the brain: insights from sign language research. Mahwah (NJ): Lawrence Erlbaum and Associates; 2002.

[19] Farah M, Wilson K, Drain H, Tanaka J. The inverted face inversion effect in prosopagnosia: evidence for mandatory, face-specific perceptual mechanisms. Vision Research 1995;35:2089-93.

[20] Frank RJ, Damasio H, Grabowski TJ. Brainvox: an interactive, multimodal visualization and analysis system for neuroanatomical imaging. Neuroimage 1997;5:13-30.

[21] Friston KJ, Holmes AP, Worsley KJ, Poline J-B, Frith CD, Frackowiak RSJ. Statistical parametric maps in functional imaging: a general linear approach. Human Brain Mapping 1995;2:189-210.

[22] Gorno-Tempini ML, Prince CJ, Josephs O, Vandenberghe R, Cappa $\mathrm{SF}$, Kapur N, et al. The neural systems sustaining face and proper-name processing. Brain 1998;121:2103-18.

[23] Grabowski TJ, Damasio H, Frank R, Hichwa R, Boles Ponto LL, Watkins GL. A new technique for PET slice orientation and MRI-PET co-registration. Human Brain Mapping 1995;2:123-33.

[24] Grabowski TJ, Frank RJ, Brown CK, Damasio H, Boles Ponto LL, Watkins GL, et al. Reliability of PET activation across statistical methods, subject groups, and sample sizes. Human Brain Mapping 1996;4:23-46.

[25] Grabowski TJ, Damasio H, Tranel D, Ponto LLB, Watkins GL, Hichwa RD, et al. Physiologic correlates of retrieving names of non-unique concrete entities. Society for Neuroscience Abstracts 2000;26:1251.

[26] Grabowski TJ, Damasio H, Tranel D, Ponto LLB, Hichwa RD, Damasio AR. A role for left temporal pole in the retrieval of words for unique entities. Human Brain Mapping 2001;13:199-212.

[27] Hickok G, Bellugi U. The signs of aphasia. In: Boller F, Grafman $\mathrm{J}$, series editors, Berndt RS, volume editor. The handbook of neuropsychology: language and aphasia, vol. 2. Amsterdam: Elsevier; 2001. p. $31-50$

[28] Holmes CJ, Hoge R, Collins L, Woods RP, Evans AC, Toga AW. Enhancement of MR images using registration for signal averaging. Journal of Computer Assisted Tomography 1998;22:324-33.
[29] van der Hulst H. Units in the analysis of signs. Phonology 1993;10:209-41.

[30] Indefrey P, Levelt WMJ. The neural correlates of language production. In: Gazzaniga M, editor. The new cognitive neurosciences, Cambridge (MA): MIT Press; 2000. p. 845-65.

[31] Kucera H, Francis WN. Computational analysis of present-day American english. Providence (RI): Brown University Press; 1967.

[32] Levelt WMJ. Speaking: from intention to articulation. Cambridge (MA): MIT Press; 1989

[33] Liddell S, Johnson R. American Sign Language: the phonological base. Sign Language Studies 1989;64:197-277.

[34] McCullough S, Emmorey K. Face processing by deaf ASL signers: evidence for expertise in distinguishing local features. Journal of Deaf Studies and Deaf Education 1997;2(4):212-22.

[35] Padden C. The ASL lexicon. Sign Language Linguistics 1998; 1(1):39-60

[36] Poizner H, Klima ES, Bellugi U. What the hands reveal about the brain. Cambridge: MIT Press; 1987.

[37] Rossion B, Dricot L, Devolder A, Bodart JM, Cormmelinck M, deGelder B, et al. Hemispheric asymmetries for whole-based and part-based face processing in the human fusiform gyrus. Journal of Cognitive Neuroscience 2000;12:793-802.

[38] Snyder LH, Grieve KL, Brotchie P, Andersen RA. Separate body- and world-referenced representations of visual space in parietal cortex. Nature 1998;394:887-91.

[39] Talairach J, Tournoux P. Co-planar stereotaxic atlas of the human brain. New York: Thieme; 1988

[40] Tanji J. New concepts of the supplementary motor area. Current Opinion in Neurobiology 1996;6:782-7.

[41] Tranel D, Damasio H, Damasio AR. Double dissociation between overt and covert face recognition. Journal of Cognitive Neuroscience 1995; 7:425-4332.

[42] Woods RP, Mazziotta JC, Cherry SR. MRI-PET registration with automated algorithm. Journal of Computer Assisted Tomography 1993; 17:536-46.

[43] Woods RP, Daprett M, Sicotte NL, Toga AW, Mazziotta JC. Creation and use of a Talairach-compatible atlas for accurate, automated, nonlinear intersubject registration, and analysis of functional imaging data. Human Brain Mapping 1999;8:73-9.

[44] Wilcox S. The phonetics of fingerspelling. Philadelphia: John Benjamins; 1992.

[45] Wise RJ, Greene J, Buchel C, Scott SK. Brain regions involved in articulation. Lancet 1999;353:1057-61.

[46] Worsley KJ, Evans AC, Marrett S, Neelin P. A three-dimensional statistical analysis for CBF activation studies in human brain. Journal of Cerebral Blood Flow Metabolism 1992;12:900-18.

[47] Worsley KJ. Local maxima and the expected Euler characteristic of excursion sets of c2,F, and t fields. Advances in Applied Probability 1994;26:13-42.

[48] Ziegler W, Kilian B, Deger K. A case of left supplementary motor area hemorrhage. Neuropsychologia 1997;35(9):1197-208. 